Published in final edited form as:

Ecotoxicol Environ Saf. 2020 September 15; 201: 110726. doi:10.1016/j.ecoenv.2020.110726.

\title{
Prenatal Exposure to Ambient Air Multi-Pollutants Significantly Impairs Intrauterine Fetal Development Trajectory
}

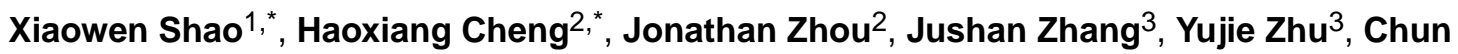 \\ Yang $^{3}$, Antonio Di Narzo ${ }^{2}$, Jing $\mathrm{Yu}^{4}$, Yuan Shen ${ }^{5}$, Yuanyuan $\mathrm{Li}^{6}$, Shunqing $\mathrm{Xu}^{6}$, Zhongyang \\ Zhang $^{2}$, Jia Chen ${ }^{7}$, Jiajing Cheng ${ }^{1, \dagger}, \mathrm{Ke} \mathrm{Hao}^{2,3,8, \dagger}$ \\ ${ }^{1}$ Department of Obstetrics and Gynecology, Shanghai Tenth People's Hospital, Tongji University, \\ Shanghai, China \\ 2Department of Genetics and Genomic Sciences, Icahn School of Medicine at Mount Sinai, New \\ York, NY, USA \\ ${ }^{3}$ Department of Respiratory Medicine, Shanghai Tenth People's Hospital, Tongji University, \\ Shanghai, China \\ ${ }^{4}$ Department of Ophthalmology, Shanghai Tenth People's Hospital, Tongji University, Shanghai, \\ China \\ ${ }^{5}$ Department of Psychiatry, Shanghai Tenth People's Hospital, Tongji University, Shanghai, China \\ ${ }^{6}$ Key Laboratory of Environment and Health, Ministry of Education \& Ministry of Ecology and \\ Environment, and State Key Laboratory of Environmental Health, School of Public Health, Tongji \\ Medical College, Huazhong University of Science and Technology, Wuhan, Hubei, China \\ ${ }^{7}$ Department of Environmental Medicine and Public Health, Icahn School of Medicine at Mount \\ Sinai, New York, NY, USA \\ ${ }^{8}$ College of Environmental Science and Engineering, Tongji University, Shanghai, China
}

\section{Abstract}

\footnotetext{
†Corresponding Authors: chengjiajing1963@163.com and ke.hao@mssm.edu.

* Contributed equally to this work

Xiaowen Shao: Conceptualization, Methodology, Data Acquisition, Haoxiang Cheng: Data analysis, Writing- Original draft preparation; Jonathan Zhou: Data Acquisition; Jushan Zhang: Data curation and results interpretation; Yujie Zhu: Data curation; Chun Yang: Data curation; Antonio Di Narzo: Data analysis; Jing Yu: Results interpretation; Yuan Shen: Results interpretation; Yuanyuan Li: Writing-Reviewing and Editing, Shunqing Xu: Writing- Reviewing and Editing, Zhongyang Zhang: Data analysis; Jia Chen: Results interpretation, Writing- Reviewing and Editing; Jiajing Cheng: Conceptualization, Methodology; Ke Hao: Conceptualization, Methodology, Supervision, Writing- Original draft preparation

Publisher's Disclaimer: This is a PDF file of an unedited manuscript that has been accepted for publication. As a service to our customers we are providing this early version of the manuscript. The manuscript will undergo copyediting, typesetting, and review of the resulting proof before it is published in its final form. Please note that during the production process errors may be discovered which could affect the content, and all legal disclaimers that apply to the journal pertain.

Competing interests

No competing interests declared

Declaration of interests

The authors declare that they have no known competing financial interests or personal relationships that could have appeared to influence the work reported in this paper.
} 
Background-Impaired in utero fetal growth trajectory may have long term health consequences of the newborns and increase risk of adulthood metabolic diseases. Prenatal exposure to air pollution has been linked to fetal development restriction; however, the impact of exposure to ambient air pollutants on the entire course of intrauterine fetal development has not been comprehensively investigated.

Methods-During 2015 - 2018, two cohorts of mother-infant dyads ( $\mathrm{N}=678$ and 227) were recruited in Shanghai China, from which three categories of data were systematically collected: (1) daily exposure to six air pollutants during pregnancy, (2) fetal biometry in the $2^{\text {nd }}$ (gestational week 24, [GW24]) and $3^{\text {rd }}$ trimester (GW36), and (3) neonatal outcomes at birth. We investigated the impact of prenatal exposure to air pollutant mixture on the trajectory of fetal development during the course of gestation, adjusting for a broad set of potential confounds.

Results-Prenatal exposure to $\mathrm{PM}_{2.5}, \mathrm{PM}_{10}, \mathrm{SO}_{2}$ and $\mathrm{O}_{3}$ significantly reduced fetal biometry at GW24, where $\mathrm{SO}_{2}$ had the most potent effect. For every $10 \mathrm{ug} / \mathrm{m}^{3}$ increment increase of daily $\mathrm{SO}_{2}$ exposure during the $1^{\text {st }}$ trimester shortened femur length by $2.20 \mathrm{~mm}(\mathrm{p}=6.7 \mathrm{E}-21)$ translating to $5.3 \%$ reduction from the average of the study cohort. Prenatal air pollution exposure also decreased fetal biometry at GW36 with attenuated effect size. Comparing to the lowest exposed quartile, fetus in the highest exposed quartile had 6.3\% ( $\mathrm{p}=3.5 \mathrm{E}-5)$ and $2.1 \%(\mathrm{p}=2.4 \mathrm{E}-3)$ lower estimated intrauterine weight in GW24 and GW36, respectively; however, no difference in birth weight was observed, indicating a rapid catch-up growth in the $3^{\text {rd }}$ trimester.

Conclusions-To our knowledge, for the first time, we demonstrated the impact of prenatal exposure to ambient air pollutants on the course of intrauterine fetal development. The altered growth trajectory and rapid catch-up growth in associated with high prenatal exposure may lead to long-term predisposition for adulthood metabolic disorders.

\section{Graphical Abstract}




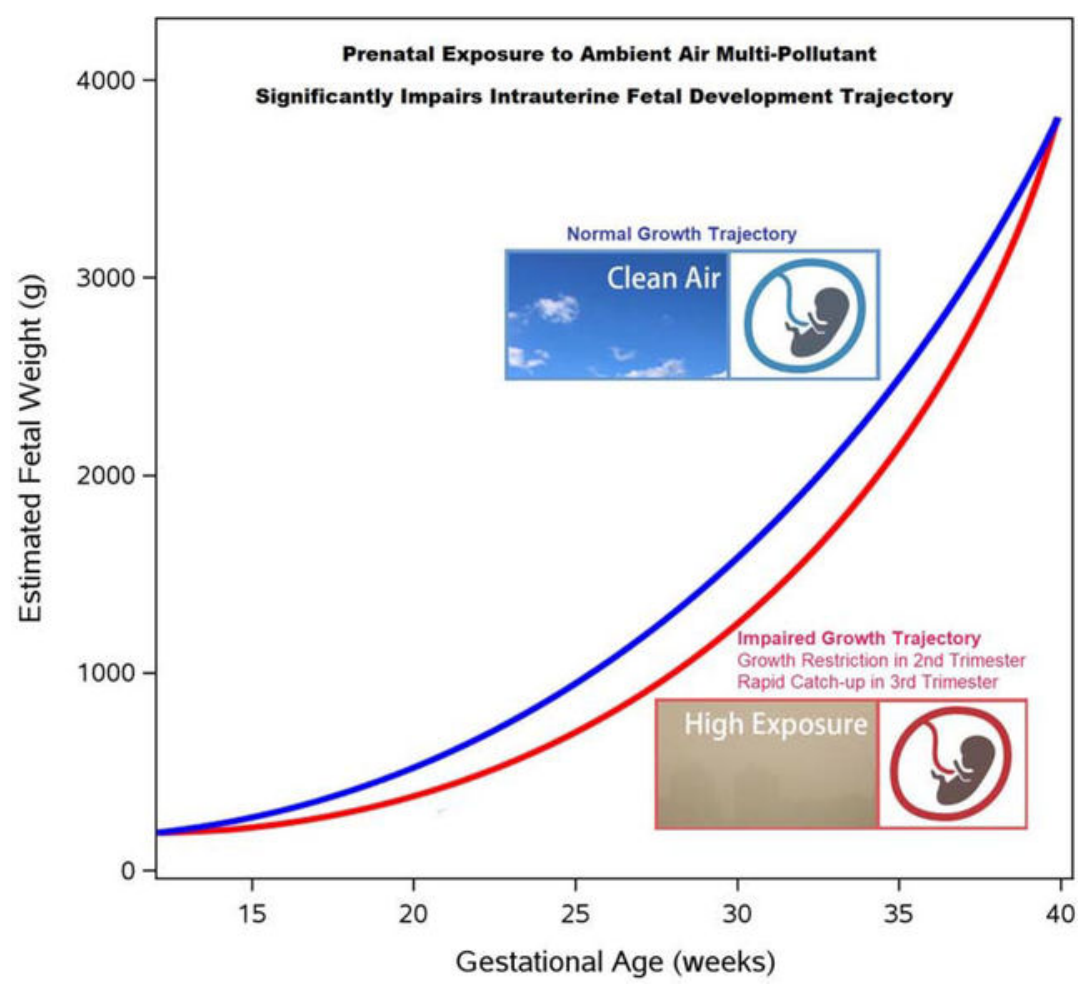

\section{Keywords}

prenatal ambient air pollutant exposure; fetal biometry; birth outcome; fetal development trajectory

\section{Introduction}

Impaired in utero fetal growth represents one of the greatest public health threats worldwide. The World Health Organization (WHO) estimated that $>20$ million LBW babies are born each year (15\% to $20 \%$ of all births), nearly $95 \%$ of them in developing countries (https:// www.who.int/nutrition/topics/globaltargets_lowbirthweight_policybrief.pdf). It is now accepted that restricted fetal growth and low birth weight $(<2500 \mathrm{~g})$ may result in rapid catch-up growth which is associated with an increased risk of multiple diseases in adulthood, including hypertension, obesity, cardiovascular diseases, diabetes, and cancers (Zheng et al., 2016). This phenomenon is referred as the Developmental Origins of Health and Diseases (DOHaD) paradigm (Barker, 1997; Barker, 2004; Barker and Godfrey, 2001). Identification of the risk factors that alter fetal growth trajectory will provide prevention opportunities towards improving children's health and reducing risk of many adult diseases. While tremendous efforts have been made to understand these risk factors, results from epidemiologic studies have been inconsistent and the environmental factors that may induce human suboptimal fetal growth and rapid catch-up growth are currently unclear.

In the recent decades, several birth cohort studies have evaluated prenatal exposure to ambient air pollutants including nitrogen dioxide $\left(\mathrm{NO}_{2}\right)$, particulate matter $(\mathrm{PM})$, and 
polycyclic aromatic hydrocarbons (PAHs) and fetal growth but yielded divergent results (Vieira, 2015). Van den Hooven et al reported prenatal $\mathrm{NO}_{2}$ and $\mathrm{PM}_{10}$ exposure reduced birth weight, birth length and head circumference, but no significant effect on gestational age (van den Hooven et al., 2012). Gehring et al found no associations between prenatal PM exposure and BW in a Dutch prospective cohort (Gehring et al., 2011). In a multicenter study conducted in Spain, $\mathrm{NO}_{2}$ exposure during pregnancy was found to reduce birth length, but benzene exposure had no effect on fetal development (Estarlich et al., 2011). A metaanalysis of 25 observational studies reported that an increase of $10 \mathrm{ug} / \mathrm{m}^{3}$ prenatal $\mathrm{PM}_{10}$ exposure was associated with $14.8 \mathrm{~g}$ decrease in birth weight (Zhu et al., 2015).

Beyond neonatal outcomes, intrauterine fetal biometry assessed by ultrasound also has been examined for association with air pollutants exposure, but most of the existing literatures focused on $\mathrm{NO}_{2}$. In a Spanish study, exposure to $\mathrm{NO}_{2}$ was inversely associated with biparietal diameter (BPD) and estimated fetus weight between weeks 20 and 32 of gestation (Iniguez et al., 2012). In the same cohort, a follow-up analysis on traffic related air pollution (TRAP) reported $\mathrm{NO}_{2}$ and aromatic hydrocarbon exposure was inversely associated with BPD growth during gestation week (GW) 20 to 32, but no relationships noted with other fetal growth parameters (Aguilera et al., 2010). The MAPSS (Maternal Air Pollution in Southern Sweden) cohort estimated negative effects for prenatal $\mathrm{NO}_{\mathrm{x}}$ exposure: decrease of GW32-33 abdominal diameter and femur length (FL) by $-0.10(-0.17,-0.03)$ and -0.13 $(-0.17,-0.01) \mathrm{mm}$, respectively, per $10 \mu \mathrm{g} / \mathrm{m}^{3}$ increment of $\mathrm{NO}_{\mathrm{x}}$ (Malmqvist et al., 2017).

China is one of the regions in the world of severe ambient air pollution (Wang et al., 2017; $\mathrm{Xu}$ et al., 2016). $\mathrm{PM}_{2.5}, \mathrm{PM}_{10}, \mathrm{SO}_{2}$ and $\mathrm{O}_{3}$ have been reported in association with risks of preterm birth in a cohort of Guangdong province, China, but was not statistically significant after multiple testing adjustment. In a Shanghai China cohort, after adjusting for other pollutants, $10 \mu \mathrm{g} / \mathrm{m}^{3}$ increment in prenatal $\mathrm{PM}_{2.5}$ exposure reduced average fetal abdominal circumference (AC), BDP and FL by $5.48 \mathrm{~mm}, 5.57 \mathrm{~mm}$ and $5.47 \mathrm{~mm}$, respectively (Cao et al., 2019), where the average biometry were measured at several gestational ages. However, the impact of prenatal $\mathrm{PM}_{2.5}$ exposure on the fetus growth trajectory during the course of gestation has not been investigated.

To date, our understanding of prenatal exposure to ambient air pollutant mixture and fetus development at various gestational stages is incomplete. Most studies only investigate selected pollutants (e.g. $\mathrm{NO}_{2}$ and $\mathrm{PM}_{2.5}$ ) and outcome at a single time point during gestation. In order to fill in such knowledge gaps, we established human cohorts in Shanghai, China and collected three categories of data (1) daily exposure to six air pollutants during pregnancy [ 3 months prior to conception - birth]; (2) fetal biometry assessed by ultrasound in the $2^{\text {nd }}$ (gestational week 24 [GW24]) and $3^{\text {rd }}$ trimester (GW36); and (3) neonatal outcomes at birth. We are positioned to characterize the impact of prenatal exposure to multiple ambient air pollutant measurements on the trajectory of in utero fetal development in a comprehensive manner. 


\section{Materials and Methods \\ Discovery cohort}

From the subjects who received obstetricians/gynecologists (OB/GYNs) care and delivered infants in the Shanghai Tenth People's Hospital during 2015-2018, we enrolled motherinfant dyads met the following inclusion/exclusion criteria into the discovery cohort. The subject resided in Shanghai, China and vicinity area three month before and throughout pregnancy. We excluded subjects with infectious disease, thyroid diseases, psychiatric disease, cognitive disorders, malignancies, severe kidney or liver dysfunction, severe or moderate preterm birth ( $<36$ week of gestational age), preeclampsia and major birth defect. We also excluded smokers and infants of assisted reproductive technology and in vitro fertilization. We excluded mothers whose pre-pregnancy body mass index $<18$. From the medical record database in the Shanghai Tenth People's Hospital, we retrieved de-identified data and preformed chart review, and obtained three batches of variables (1) mother's residential address, demographic and clinical parameters, (2) fetus biometry at GW24 and GW36 assessed by ultrasound, and (3) infant dimension measures at birth. The study was approved by the Institutional Review Boards at the Shanghai Tenth People's Hospital, Shanghai, China.

\section{Replication cohort}

In order to validate the discovery cohort findings, we obtained de-identified data of additional 227 mother-infant dyads from Shanghai Tenth People's Hospital medical record database. The inclusion/exclusion criteria and chart review methods were identical as in the discovery cohort. The infants of the replication cohort were delivered during 2015-2018, identical as in the discovery cohort. The data access and analysis were approved by the Institutional Review Boards at Shanghai Tenth People's Hospital, Shanghai, China.

\section{Assessment of fetus biometry}

Fetal biometry parameters: biparietal diameter, femur length, humerus length (HL), abdominal circumference were assessed at two time points, GW24 and GW36, by ultrasound during routine $\mathrm{OB} / \mathrm{GYN}$ visit. BPD measures the diameter of the fetus skull, femur length measures the fetal leg and humerus length measures the fetal arm length. All measurements were conducted three times, and their average were used in data analysis. After data retrieval and chart review we conducted careful data QC to verify/correct suspicious values by manually confirming the medical chart.

\section{Estimating fetal weight}

We estimate the weight of fetus, as well as the head and trunk weight, using formula published by Shinozuka et al (Shinozuka et al., 1987): fetus weight $=$ weight $_{\text {head }}+$ weight $_{\text {trunk }}=1.07 \times \mathrm{BPD}^{3}+0.3 \times \mathrm{AC}^{2} \times \mathrm{FL}$, where weight was in gram, and $\mathrm{BPD}, \mathrm{AC}$ and FL were in $\mathrm{cm}$. 


\section{Estimating ambient air pollutant exposure level}

From two sources, we obtained air pollutant concentration at each subject's residence address.

1. Ambient air pollutant data (hourly concentrations) is published by $22 \mathrm{MEE}$ (Ministry of Ecology and Environment of China) monitoring stations in Shanghai, China and vicinity area, from 2014 to 2018 (http:// beijingair.sinaapp.com/). The latitude and longitude of each station was also known. In parallel, the residential addresses of the participants were geocoded into latitude and longitude using google map, and pollutant concentration of the closest station to each individual was assigned as her exposure level. In total, 11 MEE stations were the closest station to at least one participant (Table S1 and Fig S1), and data from these stations were used in this study.

2. Real-time and high geographical granularity air quality data from UrbanAir (Zheng et al., 2013). UrbanAir compute pollutant levels based on the historical and real-time air quality data reported by monitor stations, meteorology information, traffic flow, human mobility, and structure of road networks. We map each study subject's address to a $1 \mathrm{~km}^{\prime} 1 \mathrm{~km}$ grid, and assign the UrbanAir reported pollutant concentration of the particular grid as her exposure level. We found the pollutant data from source (1) and (2) are highly consistent.

In total, six pollutants were investigated: $\mathrm{SO}_{2}, \mathrm{PM}_{2.5}, \mathrm{PM}_{10}, \mathrm{O}_{3}, \mathrm{NO}_{2}$, and $\mathrm{CO}$. We computed each participant's average exposure level of four gestational periods, preconception (PC): three months prior to conception, first trimester (T1): 0-13 week in pregnancy, second trimester (T2): 14-26 week in pregnancy, third trimester (T3): 27 week delivery.

\section{Data Analysis}

All data analysis was conducted using R statistical software (version 3.6.2). Pearson correlation were computed among pollutants and among fetus/newborn biometry. Hierarchical clustering was performed using hclust function in R.

First, we tested the impact of prenatal air pollution exposure to fetus development in the discovery cohort, second, we validated results in replication cohort using identical models. In detail, we fit robust linear model (rlm): $Y=E+Z$ (Model 1), where Y denotes the fetus/ infant biometry of interest and $E$ denotes the exposure of a given pollutant. $Z$ denotes covariables including mother's age, height, occupation, birth calendar quarter (ie, $1^{\text {st }}$ quarter: Jan-Mar, $2^{\text {nd }}$ quarter: Apr-Jun, $3^{\text {rd }}$ quarter: Jul-Sep, $4^{\text {th }}$ quarter: Oct-Dec), gravidity, parity, method of delivery, and exposure to other pollutants. These covariables were selected based on literature and plausible biological relevance. The model was fit for each outcome pollutant - gestational period combination separately. Given the six pollutants formed two clusters (Fig S2) and the Cluster 1 pollutants $\left(\mathrm{SO}_{2}, \mathrm{PM}_{2.5}, \mathrm{PM}_{10}, \mathrm{NO}_{2}\right.$, and $\left.\mathrm{CO}\right)$ were highly correlated (ie, co-linearity), we constructed principle components for Cluster 1 in each of the gestational period (PC, T1, T2 and T3), where first principle component explained 
$79 \%-83 \%$ variability. In testing the effect of Cluster 1 pollutant, we adjusted $\mathrm{O}_{3}$ in the model, and in testing the effect of $\mathrm{O}_{3}$, we adjusted first principle component of Cluster 1 .

\section{Aggregated relative potency (ARP)}

ARP is a weighted sum of the concentration of multiple pollutants, where weights are assigned as coefficients in Model 1. In this sense, ARP can be viewed as the aggregated biological effect of the pollutant mixture. This flexible technique allows weights to vary in different development stages (e.g., PC, T1, T2 and T3) and towards different fetal biometry. Specifically, the ARP was computed as

$$
\mathrm{ARP}=\Sigma_{i}\left(\text { pollutant }_{i} \times \beta_{i}\right)
$$

[Model 2]

, where $\beta_{i}$ denote the association coefficient between pollutant $t_{i}$ and fetal biometry, estimated in Model 1

\section{Results}

\section{Ambient air pollutant level}

From two sources, we obtained hourly levels of 6 pollutants from 2015 - 2018 (Materials and Methods), and the average pollutant concentration during study period were summarized in Table S1 and S2. The PM monthly average ranged between 29.48 and $64.79 \mathrm{ug} / \mathrm{m}^{3} 2.5$; and $\mathrm{PM}_{10}$ monthly average ranged between 46.32 and $88.63 \mathrm{ug} / \mathrm{m}^{3}$ (Table S1 and S2). The pollutant levels showed both spatial and temporal patterns. In general, the air quality was better in the east part than the west part of the city. Focused on the monitoring stations which is closest to at least one patient (11 stations in total, Table $\mathrm{S} 1$ ), the average $\mathrm{PM}_{10}$ and $\mathrm{PM}_{2.5}$ levels of the stations east of Huangpu river were $8.4 \%$ and $6.3 \%$ lower, respectively, than the stations west of Huangpu river during study period (Table S1 and S2). Meanwhile, the daily average pollutant levels of the 11 MEE stations were highly correlated (Fig S3). Such spatial correlation was more substantial in particulate matters than in gas pollutants. For example, the mean correlation of PM2.5 among stations were 0.95 , and mean correlation of CO among stations were 0.72 .

Clear temporal pattern is also observed, all pollutants except $\mathrm{O}_{3}$ had higher concentration in the $1^{\text {st }}$ and $4^{\text {th }}$ calendar quarters than the other two quarters, in contrast, $\mathrm{O}_{3}$ showed reverse pattern (Table S2 and Fig 1). The concentration of $\mathrm{PM}_{2.5}, \mathrm{PM}_{10}, \mathrm{CO}$ and $\mathrm{SO}_{2}$ trended downward consistently from 2015 to 2018 (Fig 1), primarily attributable to increasingly stringent clean air policy in China (Zhang et al., 2019). However, the concentration of $\mathrm{NO}_{2}$ and $\mathrm{O}_{3}$ were largely unchanged during 2015-2018. Hierarchical clustering grouped the six pollutants into two clusters (Fig S3). Cluster 1 included $\mathrm{SO}_{2}, \mathrm{PM}_{2.5}, \mathrm{PM}_{10}, \mathrm{NO}_{2}$, and $\mathrm{CO}$ where the mean inter-pollutant correlation was 0.72 . Cluster 2 contained $\mathrm{O}_{3}$, which had negative correlation with cluster 1 pollutants.

\section{Demographic and clinical characteristics}

A total of 678 and 227 mother-infant dyads were recruited to the discovery and replications cohorts, respectively. The two cohorts are comparable in terms of fetal/neonatal biometry 
(Table 1) and maternal characteristics (Table S3). The replication had more female (55.1\%) than in discovery cohort (45.6\%), and had slighted smaller head circumference: $33.6 \mathrm{~cm}$ vs. $33.8 \mathrm{~cm}, \mathrm{p}=0.005$ (Table 1). The mothers' average age at delivery was 29.0 and 29.2 years in the discovery and replication cohorts. $64.9 \%$ and $63.9 \%$ of the delivery in discovery and replication cohorts were vaginal (Table S3).

We pooled the cohorts in hierarchical clustering analysis, which revealed the correlation architecture among the fetus and infant biometry (Fig 2). The traits measured at each gestational stage (GW24, GW36 and birth) grouped into clusters. For example, GW24 biometry (BDP, FL, HL and AC) formed a cluster, and so did the GW36 biometry. Importantly, gestational age is negatively correlated with GW24 and GW36 biometry, indicating prolonged gestation could be a mechanism to compensate for slow fetal growth. We restricted hierarchical clustering analysis within the 585 mother-infant dyads of vaginal delivery and the findings remained unchanged (Fig S4).

\section{Fetal development was significantly influenced by prenatal ambient air pollutant exposure}

First, we examined the association between fetal/neonatal outcomes and prenatal exposure in the discovery cohort using Model 1 (Materials and Methods). The statistical tests were conducted on each outcome - pollutant - gestational period combination separately, resulted in a total of 336 tests, and false discover rate (FDR) was calibrated using Benjamini and Hochberg procedure (Benjamini and Hochberg, 1995) to control multiple testing (Fig 3A and Table S4). Exposure to $\mathrm{PM}_{2.5}, \mathrm{PM}_{10}$, and $\mathrm{SO}_{2}$ during $\mathrm{PC}, \mathrm{T} 1$ and $\mathrm{T} 2$ periods significantly reduced GW24 FL, HL and AC (Fig 3A). For example, a $10 \mathrm{ug} / \mathrm{m}^{3}$ increment of $\mathrm{PM}_{2.5}$ exposure during the first trimester reduced FL by $0.839 \mathrm{~mm}(\mathrm{p}=5.1 \mathrm{E}-12$ and $\mathrm{FDR}=3.5 \mathrm{E}-10)$; a $10 \mathrm{ug} / \mathrm{m}^{3}$ increment of $\mathrm{PM}_{10}$ exposure during the first trimester reduced FL by $0.605 \mathrm{~mm}$ ( $\mathrm{p}=1.0 \mathrm{E}-15$ and $\mathrm{FDR}=2.1 \mathrm{E}-13$ ) (Table $\mathrm{S} 4) . \mathrm{SO}_{2}$ showed the most profound effect size: a $10 \mathrm{ug} / \mathrm{m}^{3}$ increase during $\mathrm{T} 1$ reduced FL by $2.11 \mathrm{~mm}(\mathrm{p}=2.3 \mathrm{E}-15$ and $\mathrm{FDR}=2.5 \mathrm{E}-13$ ), translating to $5 \%$ reduction from the average of the study cohort. In addition, pre-conception exposure to $\mathrm{O}_{3}$ shortened GW24 FL and HL (FDR= 2.6E-3 and 4.7E-2, respectively). Exposure to air pollutant during all four gestational periods reduced GW36 FL and HL. Even a $10 \mathrm{ug} / \mathrm{m}^{3}$ increment of $\mathrm{PM}_{2.5}, \mathrm{PM}_{10}, \mathrm{SO}_{2}$ and $\mathrm{O}_{3}$ exposure during pre-conception period reduced FL by $0.474 \mathrm{~mm}$ (FDR=1.9E-4), $0.246 \mathrm{~mm}$ ( $\mathrm{FDR}=2.6 \mathrm{E}-4), 0.898 \mathrm{~mm}(\mathrm{FDR}=2.8 \mathrm{E}-4)$, and $0.240 \mathrm{~mm}(\mathrm{FDR}=2.9 \mathrm{E}-2)$ respectively, suggesting pollutant exposure may have long lasting effect during pregnancy. $\mathrm{PM}_{2.5}, \mathrm{PM}_{10}$, and $\mathrm{SO}_{2}$ exposure were positively associated with gestational age, for example a $10 \mathrm{ug} / \mathrm{m}^{3}$ increment of $\mathrm{PM}_{2.5}$ exposure during the third trimester increased gestation by 0.924 days $(\mathrm{FDR}=0.02)$. Importantly, prenatal ambient air pollutant exposure was not associated with birth weight (Fig 3A).

Second, we successfully validated the initial findings in the replication cohort (Fig 3B and Table S5). Prenatal exposure to $\mathrm{PM}_{2.5}, \mathrm{PM}_{10}, \mathrm{SO}_{2}$ and $\mathrm{O}_{3}$ reduced $\mathrm{FL}, \mathrm{HL}$ and $\mathrm{AC}$ at GW24, reduced FL and HL at GW36, and elongated gestation (Fig 3B and Table S5).

Third, we pooled the two cohorts and conducted joint analysis (Fig 3C and Table S6). Prenatal exposure to $\mathrm{PM}_{2.5}, \mathrm{PM}_{10}, \mathrm{SO}_{2}$ and $\mathrm{O}_{3}$ significantly reduced GW24 FL, $\mathrm{HL}$ and AC, where $\mathrm{SO}$ had the most potent effect. A $10 \mathrm{ug} / \mathrm{m}^{3} 2$ increment of daily $\mathrm{SO}_{2}$ exposure during 
T1 shortened FL by $2.20 \mathrm{~mm}$ (FDR=6.4E-19) translating to $5.3 \%$ reduction from the average of the study cohort. A $10 \mathrm{ug} / \mathrm{m}^{3}$ increment of $\mathrm{PM}_{2.5}$ daily exposure during $\mathrm{T} 1$ shortened FL by $0.85 \mathrm{~mm}$ (FDR=6.7E-14). Importantly, air pollution did not affect BPD (fetal skull development), pointing to asymmetric growth restriction (fetus concentrates nutrition to head growth at the expense of trunk development) (Delpisheh et al., 2008; Sharma et al., 2016a; Sharma et al., 2016b). At GW36, FL and HL were significantly associated with prenatal $\mathrm{PM}_{2.5}, \mathrm{PM}_{10}, \mathrm{SO}_{2}$ and $\mathrm{O}_{3}$ exposure. A $10 \mathrm{ug} / \mathrm{m}^{3}$ increment of daily exposure to $\mathrm{SO}_{2}$ and $\mathrm{PM}_{2.5}$ during $\mathrm{T} 1$ shortened $\mathrm{FL}$ by $1.07 \mathrm{~mm}(\mathrm{FDR}=8.4 \mathrm{E}-5)$ and $0.536 \mathrm{~mm}$ (FDR=7.2E-6), respectively (Table S6). Prenatal exposure to air pollution had no effect on birth weight, but significantly associated with longer gestation, implying a lower growth rate (Fig 3).

\section{Impact of prenatal ambient air pollutant on fetal growth trajectory}

Since each subject was exposed to multiple types of pollutants (i.e, mixture exposure), we computed the ARP (Materials and Methods), a strategy commonly used in the mixture exposure modeling. Afterwards, we ranked the infants based on the his/her ARP and divided the infants into four equal-sized quartiles. Using the lowest exposed quartile as the reference group, fetus in the highest exposed quartile had 6.3\% smaller estimated fetal weight $(\mathrm{p}=3.5 \mathrm{E}-5)$ and $7.9 \%$ smaller fetal trunk weight ( $\mathrm{p}=7.3 \mathrm{E}-9)$ at GW24. Interestingly, estimated fetal head weight showed no difference between the lowest and the highest exposed quartiles (Fig 4 and Table S7), indicating an asymmetrical growth restriction and possible placental insufficiency mechanism (Delpisheh et al., 2008; Sharma et al., 2016a; Sharma et al., 2016b). The estimated fetal weights of the two quartiles were still significantly different at GW36 with somewhat attenuated magnitude, where the highest exposed quartile had $2.1 \%(\mathrm{p}=2.4 \mathrm{E}-3)$ and $2.7 \%(\mathrm{p}=3.3 \mathrm{E}-4)$ lower body weight and trunk weight, respectively. The highest exposed quartile had 1.4 day longer gestation $(\mathrm{p}=0.0186)$, but no significant difference in birth weight comparing to lowest exposed quartile, indicating a strong catch-up growth in the third trimester. To avoid the potential confounding of delivery methods, we repeated analysis only in infants of vaginal delivery and the findings remained unchanged (Table S8).

\section{Discussions}

In the past two decades, multiple epidemiology studies have linked prenatal air pollution exposure and restricted fetus development. However, most of these studies have been conducted in Europe and North American, where the pollutant levels are much lower than in China. Further, the existing studies only examined selected pollutants (e.g. $\mathrm{NO}_{2}$ ) or at a single time point (e.g. at birth). To our knowledge, we are the first to simultaneously study multiple ambient air pollutants and full course of fetal development and fetal size biometric parameters. Key findings of this study:

1. Prenatal exposure to $\mathrm{PM}_{2.5}, \mathrm{PM}_{10}, \mathrm{SO}_{2}$ and $\mathrm{O}_{3}$ significantly reduced $\mathrm{GW} 24 \mathrm{FL}$, $\mathrm{HL}$ and $\mathrm{AC}$, where $\mathrm{SO}_{2}$ had the most potent effect. Also at GW36, FL and $\mathrm{HL}$ were significantly shortened by prenatal $\mathrm{PM}_{2.5}, \mathrm{PM}_{10}, \mathrm{SO}_{2}$ and $\mathrm{O}_{3}$ exposure. Prenatal exposure to air pollution had no effect on birth weight, but significantly associated with longer gestation, and implying slower fetal growth rate (Fig 3C). 
2. Comparing to the lowest exposed quartile, fetus in the highest exposed quartile had $6.3 \%(\mathrm{p}=3.5 \mathrm{E}-5)$ and $2.1 \%(\mathrm{p}=2.4 \mathrm{E}-3)$ lower estimated weight in GW24 and GW36, respectively, but no difference in birth weight, indicating a rapid catch-up growth in the $3^{\text {rd }}$ trimester (Fig 4).

3. The air pollution induced fetal growth restriction is strongly asymmetrical, where fetal trunk was affected more profoundly than fetal head (Fig 3 and Fig 4). The asymmetrical growth restriction may be caused by fetus preserves nutrition to head growth at the expense of trunk development in the scenario of placental insufficiency (Delpisheh et al., 2008; Sharma et al., 2016a; Sharma et al., 2016b).

Our study characterized the impact of prenatal air pollutant exposure on the trajectory of in utero development: growth restriction during early-middle stages of pregnancy, followed by rapid catch-up during late pregnancy. Vast literature linked postnatal catch-up growth with increased risk of adulthood, including hypertension, obesity, cardiovascular diseases, diabetes, and cancers (Adair et al., 2013; Mangel and Munch, 2005; Singhal, 2017; Singhal and Lucas, 2004; Zheng et al., 2016). Such association has been seen in infants in both lowand high-income countries, in infants born preterm or at term, and those born with normal or low birth weight for gestation (Singhal, 2017). Randomized trials of breast- and formula-fed infants support a causal link between early growth acceleration and infant nutrition and later risk of obesity. However, the existing literature focused on catch-up growth during early postnatal periods (e.g. the first 6 month of life). The intrauterine catch-up growth, especially during the $3^{\text {rd }}$ trimester, and its health consequences has not been investigated. It is partially due to accessibility hurdles, where fetal biometrics have to be estimated from systematic ultrasound measures. This paper reports air pollutant exposure restricts fetal growth during early pregnancy (ie, up to $2^{\text {nd }}$ trimester) and leads to growth acceleration in the $3^{\text {rd }}$ trimester. Future long-term birth cohort studies, with prenatal biometry data, are crucial to examine the association between intrauterine catch-up growth and adulthood metabolic disease risk. If such association exists, our findings will be of great public health importance that strategies to optimize the fetus growth trajectory could make a major contribution to prevent the current global epidemic of obesity and metabolic diseases (Singhal, 2017).

In China and most parts of the world, a mix of ambient air pollutants that come from different sources or are created from different chemical reactions in the atmosphere. Critical science is being conducted to manage air quality in a more integrated manner to take into account emissions and exposures of multiple pollutants in the atmosphere, rather than single pollutants (Olstrup et al., 2019; Tajudin et al., 2019; Tanzer et al., 2019; Vedal and Kaufman, 2011). We examined 6 pollutants which clearly formed two clusters (Fig S2). The Cluster 1 pollutants $\left(\mathrm{SO}_{2}, \mathrm{PM}_{2.5}, \mathrm{PM}_{10}, \mathrm{NO}_{2}\right.$, and $\left.\mathrm{CO}\right)$ were highly correlated. The first principle component Cluster 1 explained 79\%-83\% variability in the 4 pregnancy periods. From the mathematical viewpoint, such high co-linearity among variables make it very challenging to identify the pollutant-specific effect even with advanced mixture modeling methods (Bobb et al., 2015; Czarnota et al., 2015). In this paper, we presented the effect of each pollutant separately (Fig 3), and future studies, e.g. animal model under controlled exposure, are necessary to dissect the pollutant specific and synergistic effects. 
This study considered and controlled for an extensive set of potential confounders, including mother's age, height, occupation, birth calendar quarter (i.e, seasonal effect), gravidity, parity, method of delivery, and exposure to other pollutants. Cigarette smoking is known to restrict fetal growth (Pringle et al., 2005; Shisler et al., 2017), and we excluded smokers from cohort enrollment. In data QC and cleaning study, suspicious values were checked/reconfirmed by manual chart review. The statistical analysis employed robust linear model (rlm) which is resistant to extreme data point and ensure valid results. Mother-infant dyads of GA $<36$ week were excluded during data acquisition, because preterm birth is associated with many potential confounders, e.g., infection and abnormal uterus. In our cohorts, over $35 \%$ of participants were caesarean section (Table S3), which could bias the gestational age and neonatal outcomes (e.g. birth weight) estimations. We repeated all analyses only among mother-infant dyads of vaginal delivery, and results were unchanged.

In an epidemiological study, we are not in a position to directly determine the biological mechanisms on how air pollution impairs fetal growth. Nevertheless, our observations provide plausible leads towards the etiological mechanisms. Exposure to air pollution, especially fine particles, has been shown to induce oxidative stress and inflammation (Malmqvist et al., 2017; Terzano et al., 2010). Pregnant women have an approximately 50\% increased alveolar ventilation rate (Hackley et al., 2007) resulting in an increased uptake of inhaled pollutants including ultrafine particles. When inhaled, fine particles can penetrate the alveolar wall and enter the maternal bloodstream such that particles and inflammatory mediators may reach the placenta and the fetus (Thompson, 2018; Valavanidis et al., 2008). Further, placental mitochondrial DNA content has been suggested as a mediator between air pollution and birth weight (Clemente et al., 2016). It is well documented that cigarettes smoking causes placental insufficiency and in turn fetal growth restriction, and such restriction is often asymmetrical. In this study, we observed strong asymmetrical growth restriction at GW24 and GW36, where fetal trunk was affected more profoundly than fetal head. It is likely the fetus preserved nutrition to head growth at the expense of trunk development, pointing etiological mechanism that prenatal air pollution exposure impaired placenta function and constrained fetus access to nutrition (Delpisheh et al., 2008; Sharma et al., 2016a; Sharma et al., 2016b). The asymmetric growth restriction observed in this study is unlikely caused by maternal hypertension, where we tested and found the association between maternal blood pressure (Table S3) was not significantly associated with air pollution exposure. Preeclampsia was excluded during data acquisition to avoid the potential confounding effect, therefore, we are not able to examine whether air pollution increases preeclampsia risk, which is certainly an important direction of future research. BPD at GW36 was positively associated with $\mathrm{PM}_{2.5}$ exposure level in $\mathrm{T} 1$ and $\mathrm{NO}_{2}$ level in $\mathrm{PC}$ in the discovery cohort, but not replicated (Table S5). Future study would be needed to either rule out such association or confirm that early prenatal exposure to $\mathrm{PM}_{2.5}$ and $\mathrm{NO}_{2}$ increase BPD at GW36.

As another limitation, the current study did not investigate the composition of $\mathrm{PM}_{2.5}$ and $\mathrm{PM}_{10}$, which are both complex mixtures of many types of toxins. In Shanghai China, the $\mathrm{PM}_{2.5}$ mainly comprises organic matter (OM, $\left.24.9 \%\right), \mathrm{SO}_{4}{ }^{2-}(19.9 \%)$ and $\mathrm{NO}_{3}{ }^{-}(17.4 \%)$; and also contains mineral dust, $\mathrm{NH}_{4}{ }^{+}$, elemental carbon (EC) and $\mathrm{Cl}^{-}$(Liu et al., 2018). OM and EC categories were also consisted of a large variety of compounds (Feng et al., 2006), 
such as polycyclic aromatic hydrocarbon (PAH) (Feng et al., 2006). Air pollutant levels also followed a seasonal pattern (Fig 1). In northern China and north-eastern China, the wintertime peak values of $\mathrm{PM}_{2.5}$ were mainly attributed to the combustion of fossil fuels and biomass burning for domestic heating (Liu et al., 2018). In south-eastern China, where Shanghai is located, the effect of domestic heating is not as important as it is in northern China, nevertheless, the weakened diffusion and transport of pollutants from the north in the winter is more profound than other seasons (Liu et al., 2018). Future studies leveraging the $\mathrm{PM}_{2.5}$ and $\mathrm{PM}_{10}$ granular composition will be of great interest.

This study collected a rather complex dataset: longitudinal outcome (e.g., at GW24, GW36 and birth), repeated exposure measures (daily average of pollutant level) and multiple pollutant, which presented a challenge in data analysis. We applied a relatively simple strategy by pooling the daily average of pollutant level into four gestational periods and fitting multivariate regression models. However, since the pollutant levels among the gestational periods were highly correlated, our model cannot conclude with certainty which period(s) is susceptible to pollution exposure. Alternative analysis strategies include distributed lag model (DLM), which was applied to study prenatal $\mathrm{PM}_{2.5}$ exposure and childhood asthma risk (Lee et al., 2018). However, in the context of high autocorrelation of repeated measures, DLM is subjected to unreliable coefficient estimates with large variances and standard errors. Five pollutants, $\mathrm{SO}_{2}, \mathrm{PM}_{2.5}, \mathrm{PM}_{10}, \mathrm{NO}_{2}$, and $\mathrm{CO}$ were highly correlated (Fig S2). Our analysis cannot rule out the possibility that a pollutant in significantly association with fetal biometry actually has no biological effect, but simply a surrogate of the causal pollutant. In the other hand, including all the five correlated pollutants into the regression model will cause collinearity and unstable coefficient estimation. Mixture modeling, e.g., weighted quantile sum (WQS) regression (Czarnota et al., 2015) and Bayesian kernel machine regression (BKMR) (Bobb et al., 2015), can also be applied, however WQS and BKMR aim to assess the effect of pollution mixture and do not directly quantify the effect of each individual pollutant. Animal model could be a powerful solution to dissect the effect of each pollutant, where pregnant animals are exposed in a wellcontrolled manner. Fetal growth metrics can be directly measured by sacrificing the animal at the gestational stage of interest, and maternal and fetal tissues are readily available for studying etiological mechanisms (e.g. placenta insufficiency).

In summary, for the first time, we systematically studied the impact of prenatal exposure to six ambient air pollutants on fetal growth during the course of gestation. The study used two cohorts and convincingly showed that prenatal exposure to $\mathrm{PM}_{2.5}, \mathrm{PM}_{10}, \mathrm{SO}_{2}$ and $\mathrm{O}_{3}$ reduced GW24 and GW36 fetal biometry, but was not associated with birth weight, suggesting rapid catch-up at the late stage of pregnancy, which may predispose the infants to adulthood metabolic diseases. Moreover, the fetal growth restriction is also asymmetric, suggesting plausible etiological mechanism through placental insufficiency. From a public health perspective, our results provided much need evidence of prenatal air pollutant mixture exposure and impaired fetal development trajectory, and guidance in preventing both fetal growth restriction and long term health consequences. 


\section{Supplementary Material}

Refer to Web version on PubMed Central for supplementary material.

\section{Acknowledgements}

This work is partially supported by National Natural Science Foundation of China (Grant No. 21477087, 91643201, 21876134), the Ministry of Science and Technology of China (Grant No. 2016YFC0206507) and NIH-NIEHS (1R01ES029212-01).

\section{Abbreviations}

$\begin{array}{ll}\text { BPD } & \text { biparietal diameter } \\ \text { FL } & \text { femur length } \\ \text { HL } & \text { humerus length } \\ \text { AC } & \text { abdominal circumference } \\ \text { PC } & \text { prior to conception } \\ \text { T1 } & \text { first trimester } \\ \text { T2 } & \text { second trimester } \\ \text { T3 } & \text { third trimester } \\ \text { GA } & \text { gestational age } \\ \text { BL } & \text { length birth } \\ \text { BW } & \text { birth weight } \\ \text { HC } & \text { head circumference } \\ \text { GW } & \text { Gestational week } \\ \text { PM } & \text { Particulate matter }\end{array}$

\section{References}

Adair LS, et al., 2013 Associations of linear growth and relative weight gain during early life with adult health and human capital in countries of low and middle income: findings from five birth cohort studies. Lancet. 382, 525-34. [PubMed: 23541370]

Aguilera I, et al., 2010 Prenatal exposure to traffic-related air pollution and ultrasound measures of fetal growth in the INMA Sabadell cohort. Environ Health Perspect. 118, 705-11. [PubMed: 20103496]

Barker DJP, 1997 The fetal origins of coronary heart disease. Acta Paediatrica. 86, 78-82.

Barker DJP, 2004 The developmental origins of chronic adult disease. Acta Paediatrica. 93, 26-33.

Barker DJP, Godfrey KM, Fetal Nutrition and Cardiovascular Disease in Adult Life. Nutritional Health. Springer Science + Business Media, 2001, pp. 253-268.

Benjamini Y, Hochberg Y, 1995 Controlling the False Discovery Rate: A Practical and Powerful Approach to Multiple Testing. Journal of the Royal Statistical Society. Series B (Methodological). 57, 289-300. 
Bobb JF, et al., 2015 Bayesian kernel machine regression for estimating the health effects of multipollutant mixtures. Biostatistics. 16, 493-508. [PubMed: 25532525]

Cao Z, et al., 2019 Maternal exposure to ambient fine particulate matter and fetal growth in Shanghai, China. Environ Health 18, 49. [PubMed: 31096994]

Clemente DB, et al., 2016 Prenatal Ambient Air Pollution, Placental Mitochondrial DNA Content, and Birth Weight in the INMA (Spain) and ENVIRONAGE (Belgium) Birth Cohorts. Environ Health Perspect. 124, 659-65. [PubMed: 26317635]

Czarnota J, et al., 2015 Assessment of weighted quantile sum regression for modeling chemical mixtures and cancer risk. Cancer Inform. 14, 159-71. [PubMed: 26005323]

Delpisheh A, et al., 2008 Prenatal smoking exposure and asymmetric fetal growth restriction. Ann Hum Biol. 35, 573-83. [PubMed: 18932054]

Estarlich M, et al., 2011 Residential exposure to outdoor air pollution during pregnancy and anthropometric measures at birth in a multicenter cohort in Spain. Environ Health Perspect. 119, 1333-8. [PubMed: 21429861]

Feng J, et al., 2006 Characteristics of organic matter in PM2.5 in Shanghai. Chemosphere. 64, 1393400. [PubMed: 16442145]

Gehring U, et al., 2011 Traffic-related air pollution, preterm birth and term birth weight in the PIAMA birth cohort study. Environ Res. 111, 125-35. [PubMed: 21067713]

Hackley B, et al., 2007 Air pollution: impact on maternal and perinatal health. J Midwifery Womens Health. 52, 435-43. http://beijingair.sinaapp.com/. https://www.who.int/nutrition/topics/ globaltargets_lowbirthweight_policybrief.pdf. [PubMed: 17826705]

Iniguez C, et al., 2012 Prenatal exposure to traffic-related air pollution and fetal growth in a cohort of pregnant women. Occup Environ Med. 69, 736-44. [PubMed: 22843441]

Lee A, et al., 2018 Prenatal fine particulate exposure and early childhood asthma: Effect of maternal stress and fetal sex. J Allergy Clin Immunol. 141, 1880-1886. [PubMed: 28801196]

Liu Z, et al., 2018 Characteristics of PM2.5 mass concentrations and chemical species in urban and background areas of China: emerging results from the CARE-China network. Atmos. Chem. Phys $18,8849-8871$.

Malmqvist E, et al., 2017 Fetal growth and air pollution - A study on ultrasound and birth measures. Environ Res. 152, 73-80. [PubMed: 27741452]

Mangel M, Munch SB, 2005 A life-history perspective on short- and long-term consequences of compensatory growth. Am Nat. 166, E155-76. [PubMed: 16475079]

Olstrup H, et al., 2019 A Multi-Pollutant Air Quality Health Index (AQHI) Based on Short-Term Respiratory Effects in Stockholm, Sweden. Int J Environ Res Public Health. 16.

Pringle PJ, et al., 2005 The influence of cigarette smoking on antenatal growth, birth size, and the insulin-like growth factor axis. J Clin Endocrinol Metab. 90, 2556-62. [PubMed: 15713720]

Sharma D, et al., 2016a Intrauterine growth restriction - part 1. J Matern Fetal Neonatal Med. 29, 3977-87. [PubMed: 26856409]

Sharma D, et al., 2016b Intrauterine Growth Restriction: Antenatal and Postnatal Aspects. Clin Med Insights Pediatr. 10, 67-83. [PubMed: 27441006]

Shinozuka N, et al., 1987 Formulas for fetal weight estimation by ultrasound measurements based on neonatal specific gravities and volumes. Am J Obstet Gynecol. 157, 1140-5. [PubMed: 3318464]

Shisler S, et al., 2017 Smoking in Pregnancy and Fetal Growth: The Case for More Intensive Assessment. Nicotine Tob Res. 19, 525-531. [PubMed: 28403474]

Singhal A, 2017 Long-Term Adverse Effects of Early Growth Acceleration or Catch- Up Growth. Ann Nutr Metab. 70, 236-240. [PubMed: 28301849]

Singhal A, Lucas A, 2004 Early origins of cardiovascular disease: is there a unifying hypothesis? Lancet. 363, 1642-5. [PubMed: 15145640]

Tajudin M, et al., 2019 Risk of concentrations of major air pollutants on the prevalence of cardiovascular and respiratory diseases in urbanized area of Kuala Lumpur, Malaysia. Ecotoxicol Environ Saf. 171, 290-300. [PubMed: 30612017] 
Tanzer R, et al., 2019 Demonstration of a Low-Cost Multi-Pollutant Network to Quantify Intra-Urban Spatial Variations in Air Pollutant Source Impacts and to Evaluate Environmental Justice. Int J Environ Res Public Health. 16.

Terzano C, et al., 2010 Air pollution ultrafine particles: toxicity beyond the lung. Eur Rev Med Pharmacol Sci. 14, 809-21. [PubMed: 21222367]

Thompson JE, 2018 Airborne Particulate Matter: Human Exposure and Health Effects. J Occup Environ Med. 60, 392-423. [PubMed: 29334526]

Valavanidis A, et al., 2008 Airborne particulate matter and human health: toxicological assessment and importance of size and composition of particles for oxidative damage and carcinogenic mechanisms. J Environ Sci Health C Environ Carcinog Ecotoxicol Rev. 26, 339-62. [PubMed: 19034792]

van den Hooven EH, et al., 2012 Air pollution exposure during pregnancy, ultrasound measures of fetal growth, and adverse birth outcomes: a prospective cohort study. Environ Health Perspect. 120, 150-6. [PubMed: 22222601]

Vedal S, Kaufman JD, 2011 What does multi-pollutant air pollution research mean? Am J Respir Crit Care Med. 183, 4-6. [PubMed: 21193783]

Vieira SE, 2015 The health burden of pollution: the impact of prenatal exposure to air pollutants. Int J Chron Obstruct Pulmon Dis. 10, 1111-21. [PubMed: 26089661]

Wang J, et al., 2017 Particulate matter pollution over China and the effects of control policies. Sci Total Environ. 584-585, 426-447.

$\mathrm{Xu}$ J, et al., 2016 The meteorological modulation on PM2.5 interannual oscillation during 2013 to 2015 in Shanghai, China. Sci Total Environ. 572, 1138-1149. [PubMed: 27519324]

Zhang Q, et al., 2019 Drivers of improved PM2.5 air quality in China from 2013 to 2017. Proc Natl Acad Sci U S A. 116, 24463-24469. [PubMed: 31740599]

Zheng T, et al., 2016 Effects of Environmental Exposures on Fetal and Childhood Growth Trajectories. Ann Glob Health. 82, 41-99. [PubMed: 27325067]

Zheng Y, et al., 2013 U-Air: WhenUrban Air Quality Inference Meets Big Data. Proceedings of the 19th SIGKDD conference on Knowledge Discovery and Data Mining (KDD 2013).

Zhu X, et al., 2015 Maternal exposure to fine particulate matter (PM2.5) and pregnancy outcomes: a meta-analysis. Environ Sci Pollut Res Int. 22, 3383-96. [PubMed: 25163563] 


\section{Highlights}

- Impaired in utero fetal growth trajectory may have long term health consequences.

- Two cohorts of mother-infant dyads in Shanghai China was recruited to study prenatal exposure to air pollution on the course of intrauterine fetal development.

- Prenatal exposure to $\mathrm{PM}_{2.5}, \mathrm{PM}_{10}, \mathrm{SO}_{2}$ and $\mathrm{O}_{3}$ significantly reduced fetal biometry at gestational week (GW) 24 , where $\mathrm{SO}_{2}$ had the most potent effect. Prenatal exposure also decreased fetal biometry at GW 36 with attenuated effect size.

- Prenatal air pollution exposure results in a decrease in fetal biometry in the $2^{\text {nd }}$ gestational trimester and a rapid catch-up grow in the $3^{\text {rd }}$ trimester.

- The air pollution induced growth restriction is asymmetric, disproportionally affecting the trunk development. 


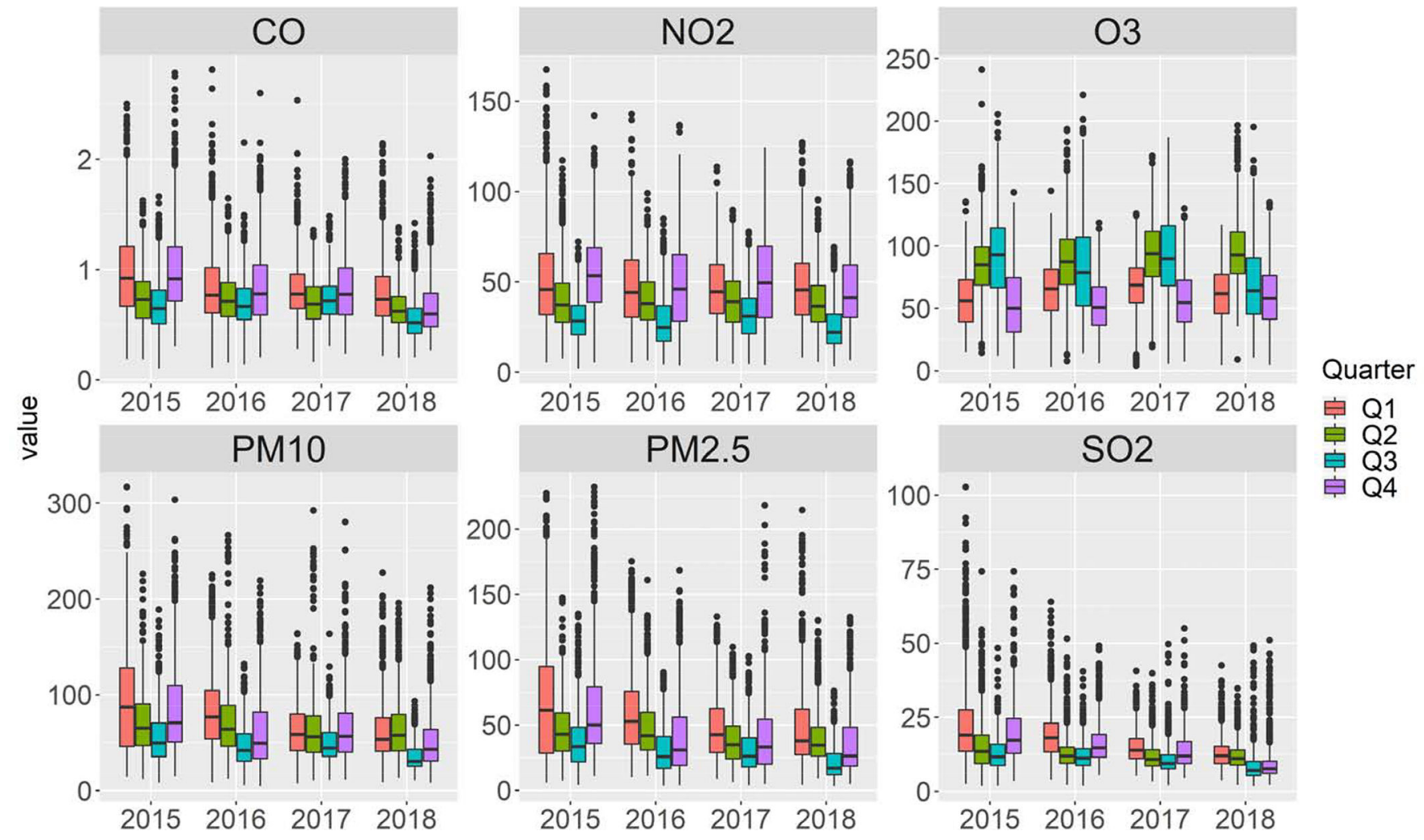

Fig 1:

Distribution of daily average of six major ambient air pollutants in Shanghai during study period by calendar quarter. 


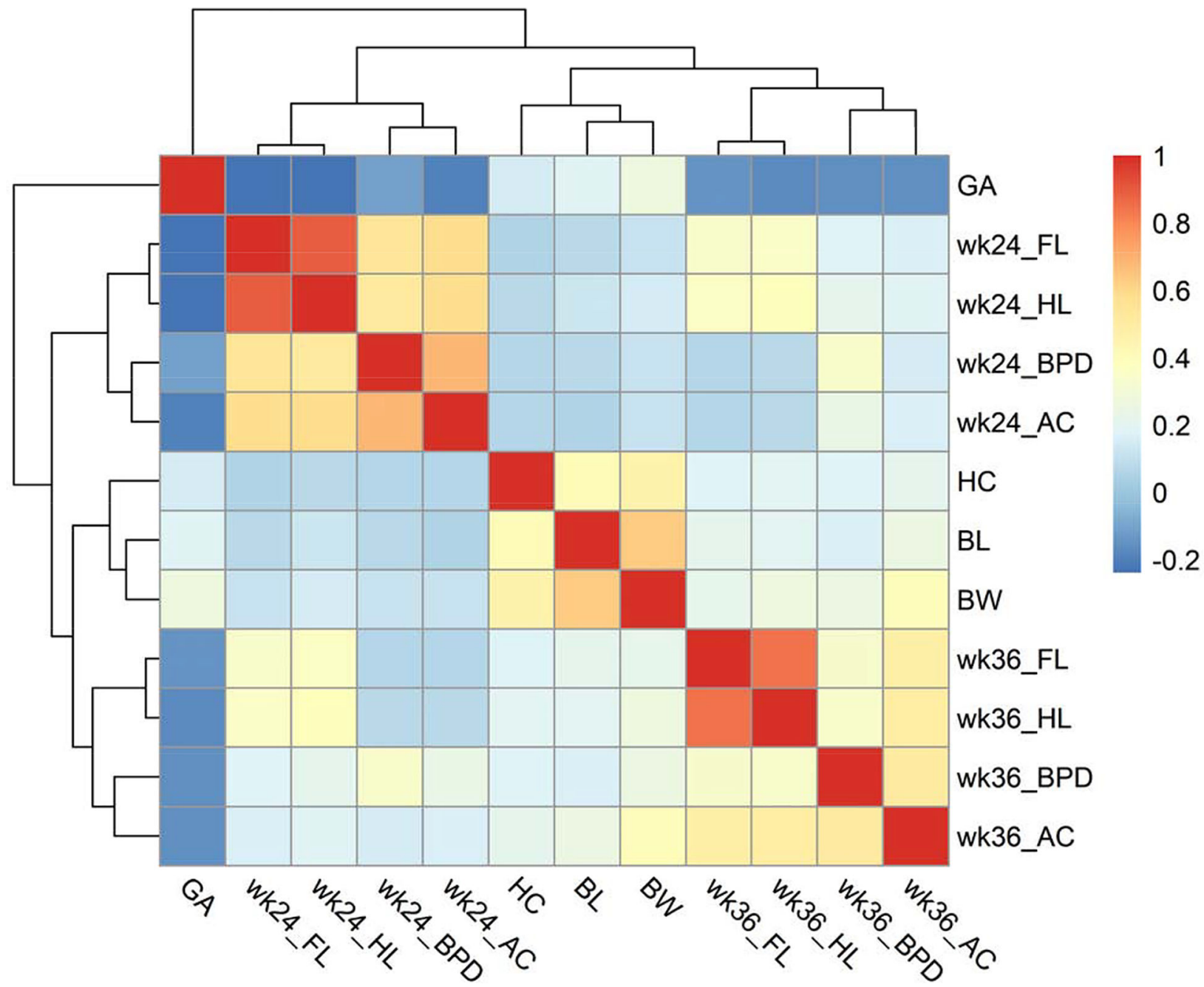

Fig 2:

Pearson correlation (r) and hierarchical clustering among fetus biometry and birth outcomes. BPD, biparietal diameter; FL, femur length; HL, humerus length; AC, abdominal circumference; GA, gestational age; BL, birth length; BW, birth weight; HC, head circumference. 
A. Discovery Cohort

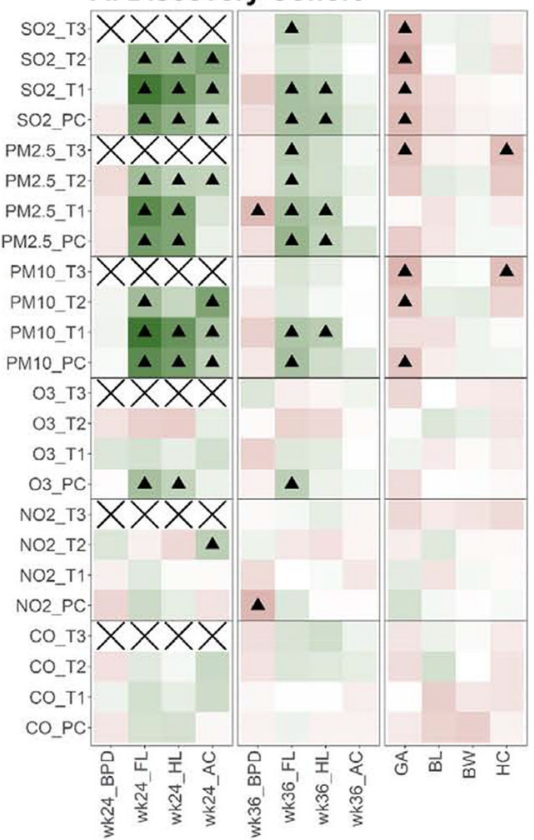

B. Replication Cohort

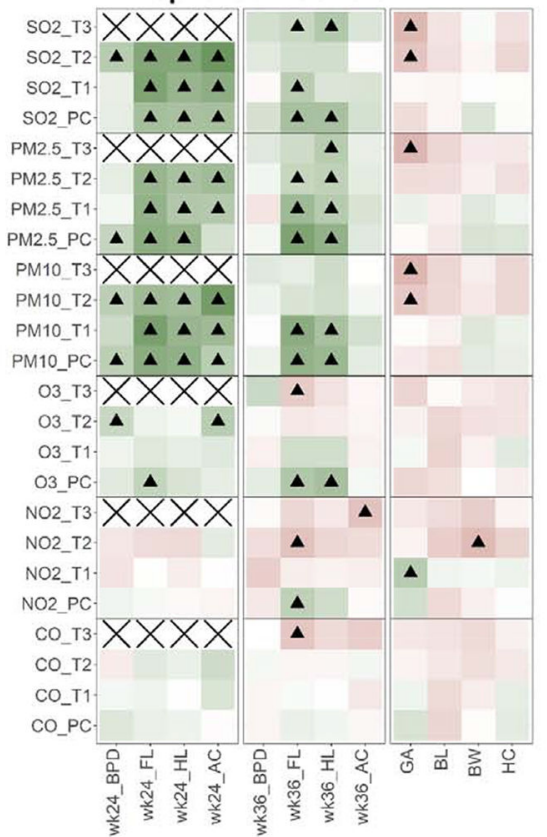

C. Pooled

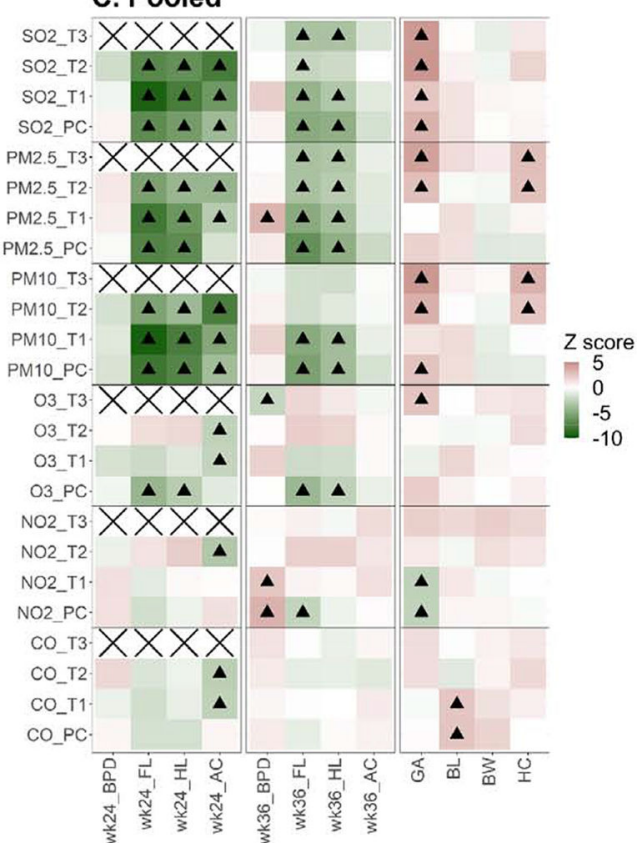

Fig 3.

Association $\mathrm{Z}$ score between ambient air pollutants during pregnancy and fetus development in discovery cohort and replication cohort. PC, three months before estimated conception time point; T1, first trimester: 0-13 week in pregnancy; T2, second trimester: 14-26 week in pregnancy; T3, third trimester: 27 week - delivery. The association test was adjusted for covariables including mother's age, mother's height, mother's occupation, birth quarter (calendar quarter), gravidity, parity, method of delivery, and exposure to other pollutants. Significant level was calibrated using Benjamini Hochberg FDR. A, Z score in discovery cohort; $\mathbf{B}, \mathrm{Z}$ score in replication cohort; $\mathbf{C}, \mathrm{Z}$ score on pooled sample (discovery and replication cohorts). In each panel, black triangle indicates FDR $\leq 5 \%$; 


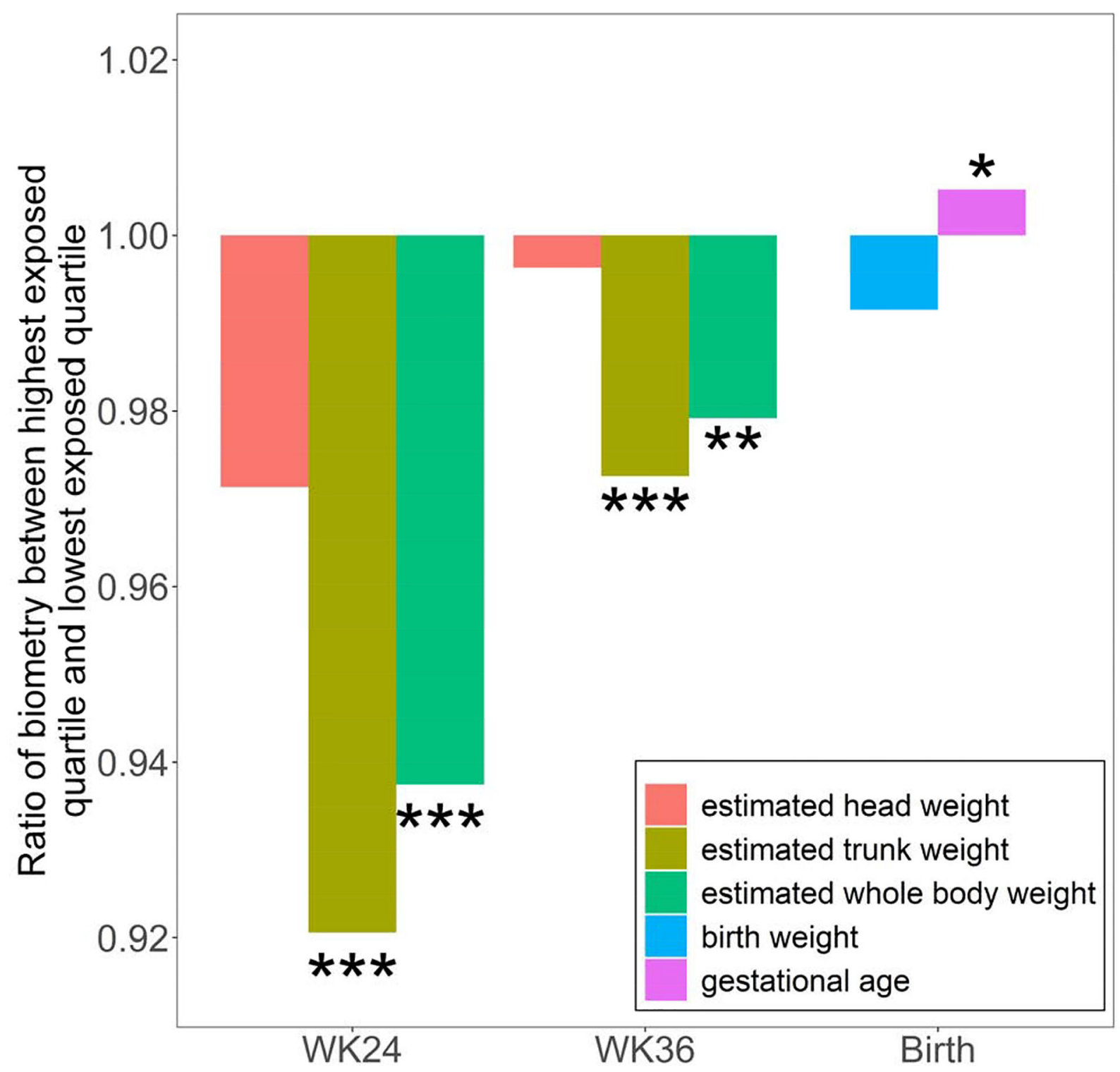

Fig 4.

Infants were ranked by prenatal exposure level, and we compared the highest exposed quartile and lowest exposed quartile (ie, reference group). Fetus weight was estimated using formula described in Materials and Methods. *: pvalue $\leq 0.05$; **: pvalue $\leq 0.01$; ***: pvalue $\leq 0.001$. 
Table 1.

Fetal biometry and infant birth outcomes

\begin{tabular}{lccc}
\hline \multicolumn{1}{c}{ Variable } & $\begin{array}{c}\text { Discovery Cohort } \\
(\mathbf{n}=\mathbf{6 7 8})\end{array}$ & $\begin{array}{c}\text { Replication Cohort } \\
(\mathbf{n}=\mathbf{2 2 7})\end{array}$ & p-value \\
\hline Infant gender (female, \%) & 45.6 & 55.1 & 0.016 \\
Week 24 fetal biometry, mm & & & \\
$\quad$ Biparietal diameter & $61.6 \pm 2.97$ & $61.2 \pm 2.97$ & 0.050 \\
$\quad$ Femur length & $41.7 \pm 2.97$ & $41.1 \pm 2.97$ & 0.214 \\
$\quad$ Humerus length & $38.7 \pm 2.97$ & $38.4 \pm 2.97$ & 0.173 \\
$\quad$ Abdominal circumference & $199.9 \pm 13.34$ & $199.8 \pm 14.83$ & 0.098 \\
Week 36 fetal biometry, mm & & & \\
$\quad$ Biparietal diameter & $90.8 \pm 2.97$ & $90.7 \pm 2.97$ & 0.383 \\
$\quad$ Femur length & $67.5 \pm 2.97$ & $67.2 \pm 2.97$ & 0.437 \\
$\quad$ Humerus length & $58.8 \pm 1.48$ & $58.6 \pm 2.97$ & 0.121 \\
$\quad$ Abdominal circumference & $317.1 \pm 8.9$ & $317.3 \pm 11.86$ & 0.825 \\
Gestational age (GA), day & $279.7 \pm 5.93$ & $280.3 \pm 7.41$ & 0.345 \\
Birth length (BL), cm & $50.1 \pm 0.0$ & $50.1 \pm 0.0$ & 0.931 \\
Birth weight (BW), gram & $3422 \pm 355.82$ & $3405.7 \pm 355.82$ & 0.578 \\
Head circumference (HC), cm & $33.8 \pm 1.48$ & $33.6 \pm 1.48$ & 0.005 \\
\hline * & & \\
In quantitative traits, mean \pm median absolute deviation (MAD) are presented. Wilcoxon rank test and Chi square tests were applied to examine \\
the differences for quantitative and categorical traits, respectively.
\end{tabular}

